\section{Iniquidades étnico-raciais nas hospitalizações por causas evitáveis em menores de cinco anos no Brasil, 2009-2014}

\author{
Ethnic and racial inequalities in hospital admissions \\ due to avoidable causes in under-five Brazilian \\ children, 2009-2014
}

\section{Inequidades étnico-raciales en las hospitalizaciones por causas evitables en menores de cinco años en Brasil, 2009-2014}

\section{Resumo}

Internacionalmente, observa-se um incremento no uso das internações por condições sensíveis à atenção primária (ICSAP) como indicador de efetividade da atenção primária à saúde. Este artigo analisa as iniquidades étnico-raciais nas internações por causas em menores de cinco anos no Brasil e regiões, com ênfase nas ICSAP e nas infecções respiratórias agudas (IRA). Com dados do Sistema de Informações Hospitalares do Sistema Único de Saúde (SIH/SUS), 2009-2014, calcularam-se proporções por causas, taxas e razões de taxas de ICSAP ajustadas por sexo e idade após a imputação múltipla de dados faltantes de cor/raça. As principais causas de internação foram doenças do aparelho respiratório (37,4\%) e infecciosas e parasitárias (19,3\%), sendo as crianças indígenas as mais acometidas. As taxas brutas de ICSAP (por 1.000) foram mais elevadas em indigenas (97,3; IC95\%: 95,3-99,2), seguidas das pardas (40,0; IC95\%: 39, 8-40,1), e as menores foram nas amarelas (14, 8; IC95\%: 14,115,5). As maiores razões de taxas ajustadas de ICSAP foram registradas entre crianças de cor/raça indígena e branca - 5,7 (IC95\%: 3,9-8,4) no país, atingindo 5,9 (IC95\%: 5,0-7,1) e 18,5 (IC95\%: 16,5-20,7) no Norte e Centro-oeste, respectivamente. As IRA permanecem como importantes causas de hospitalização em crianças no Brasil. Foram observadas alarmantes iniquidades étnico-raciais nas taxas de ICSAP, com situação de desvantagem para indígenas. São necessárias melhorias nas condições de vida, saneamento e subsistência, bem como garantia de acesso oportuno e qualificado à atenção primária à saúde das populações mais vulneráveis, com destaque para os indígenas no Norte e no Centro-oeste, a fim de minimizar iniquidades em saúde e fazer cumprir as diretrizes do SUS e da Constituição do Brasil.

Hospitalização; Saúde Infantil; Situação de Saúde dos Grupos Étnicos;

Iniquidade em Saúde; Índios Sul-Americanos
Yasmin Nascimento Farias 1

Iuri da Costa Leite 1

Marilda Agudo Mendonça Teixeira de Siqueira 2

Andrey Moreira Cardoso 1

doi: 10.1590/0102-311X00001019

\section{Correspondência}

Y. N. Farias

Escola Nacional de Saúde Pública Sergio Arouca, Fundação Oswaldo Cruz.

Rua Leopoldo Bulhões 1480, Rio de Janeiro, RJ 21041-210, Brasil.

yas.farias06@gmail.com

1 Escola Nacional de Saúde Pública Sergio Arouca, Fundação Oswaldo Cruz, Rio de Janeiro, Brasil.

2 Instituto Oswaldo Cruz, Fundação Oswaldo Cruz, Rio de Janeiro, Brasil. 


\section{Introdução}

A morbidade hospitalar é um importante componente do perfil epidemiológico, refletindo a gravidade das doenças que acometem a população, o acesso e a utilização dos serviços de saúde 1,2. Alguns agravos à saúde são particularmente sensíveis à organização da atenção primária, tendendo a existir internações reduzidas quando ações de prevenção, diagnóstico e tratamento de doenças agudas, e controle e acompanhamento das doenças crônicas são desenvolvidas de forma adequada e oportuna. As internações por essas causas têm sido chamadas de internações por condições sensíveis à atenção primária (ICSAP) e utilizadas em diversos países como um indicador de efetividade da atenção primária à saúde (APS) 2. Portanto, taxas de ICSAP elevadas em um segmento populacional sugerem limitações na cobertura e/ou na resolubilidade dos problemas de saúde na APS, evidenciando uma situação de vulnerabilidade social 3,4,5. Cada país ou região, incluindo o Brasil, busca organizar sua lista de ICSAP com base nos padrões de saúde e organização da atenção à saúde, e nos recursos e possibilidades de intervenção disponíveis 2 .

No Brasil, apesar das expressivas melhorias nos indicadores de morbimortalidade na infância 6, desigualdades regionais e étnicas em saúde infantil vêm sendo sistematicamente apontadas 7,8,9,10,11. Por exemplo, há poucos trabalhos publicados que analisam a morbidade hospitalar em crianças indígenas no país. Eles são restritos a recortes geográficos locais ou a grupos étnicos específicos, e revelam padrões de morbidade indígena compatíveis com aqueles verificados nos segmentos sociais mais vulneráveis, sobressaindo as internações por causas infecciosas, com destaque para as infecções respiratórias agudas (IRA) e diarreia, além de apresentarem elevada carga de ICSAP 12,13.

O Sistema de Informações Hospitalares do Sistema Único de Saúde (SIH/SUS) é uma importante base de dados relativa à morbidade hospitalar no Brasil, ao considerar as internações ocorridas na rede hospitalar pública e conveniada ao SUS 14, e albergar grande parte das internações hospitalares ocorridas no país (Departamento de Informática do SUS. http://tabnet.datasus.gov.br/cgi/sih/ midescr.htm, acessado em 09/Set/2018). A variável cor/raça foi inserida no SIH/SUS em 2008, possibilitando desde então análises da morbidade hospitalar com recorte étnico-racial de abrangência nacional. Embora o monitoramento das causas de hospitalização nessa perspectiva seja considerado uma ferramenta importante na investigação de iniquidades étnico-raciais em saúde 15, ela ainda é pouco explorada, particularmente no segmento infantil.

O presente estudo tem por objetivo analisar as hospitalizações de crianças menores de cinco anos no Brasil registradas no SIH/SUS no período de 2009 a 2014, segundo cor/raça, com vistas a identificar a relevância dos principais grupos de causas de internação e a importância relativa das IRA, a magnitude das taxas de ICSAP segundo cor/raça e as iniquidades étnico-raciais nos perfis das hospitalizações por causas, com ênfase nas doenças respiratórias agudas e ICSAP, agravos mais frequentes em grupos sociais mais vulneráveis da população brasileira.

\section{Material e métodos}

\section{Desenho de estudo e população}

Estudo descritivo da morbidade hospitalar por causas em crianças menores de cinco anos no Brasil e regiões. A fonte de dados usada foi o SIH/SUS, disponível em livre acesso na plataforma on-line do Departamento de Informática do SUS. Foram selecionadas todas as hospitalizações de crianças menores de cinco anos no período de 2009 a 2014 no país, sendo excluídas as Autorizações de Internações Hospitalares (AIH) de longa permanência. O recorte temporal decorre da variável cor/raça ter sido disponibilizada no SIH/SUS em 2009, e de 2014 ser o último ano com informações consolidadas no momento da análise.

\section{Causas da hospitalização}

Foram consideradas as seguintes causas de internação com base na 10a revisão da Classificação Internacional de Doenças (CID-10): (1) capítulos da CID-10; (2) internações por infecções respiratórias 
agudas (IRA) (J00-J22); (3) internações por pneumonia e influenza (P\&I) (J10-J18); e (4) internações por condições sensíveis à atenção primária (ICSAP), com base na lista brasileira 2.

A análise específica das internações por IRA e P\&I decorre do fato de estudos demonstrarem a persistência destas causas de internação em crianças no Brasil e expressiva magnitude destas causas de internação em populações vulneráveis, em particular em indígenas no país e em outras regiões do mundo 12,16 .

\section{Análise dos dados}

Realizou-se uma análise descritiva das hospitalizações, estimando-se as internações proporcionais específicas por sexo, faixa etária ( $<1$ ano e 1-4 anos), região de residência e tempo de permanência hospitalar segundo categorias de cor/raça, em dois triênios (2009-2011 e 2012-2014). Calcularam-se ainda as internações proporcionais por causas (Capítulos da CID-10; IRA; P\&I; e ICSAP) segundo cor/ raça. Em seguida, foram estimadas as taxas brutas de ICSAP com os respectivos intervalos de 95\% de confiança (IC95\%) por categoria de cor/raça, para o Brasil e regiões, após a imputação múltipla dos dados faltantes de cor/raça. Por fim, foram estimadas as razões de taxas (RT) de ICSAP ajustadas por idade e sexo, com IC95\% entre as categorias de cor/raça, para o Brasil e regiões, antes e após a imputação múltipla da variável cor/raça.

A imputação foi feita assumindo-se que os valores faltantes são aleatórios (missing at random MAR), ou seja, a probabilidade de ausência de informação em uma variável específica Y não está relacionada com os seus valores após ter sido controlada por um conjunto de variáveis supostamente associado com o mecanismo de ocorrência dos dados faltantes. Métodos para dados faltantes aleatórios estão bem documentados, e seus resultados têm sido considerados satisfatórios mesmo quando violado o pressuposto de que o mecanismo de geração de valores faltantes não esteja relacionado aos parâmetros estimados 17,18 .

Tendo em vista que as metodologias utilizadas pelo Instituto Brasileiro de Geografia e Estatística (IBGE) para estimar a população indígena foram distintas nos censos demográficos de 2000 e 2010 (https://ww2.ibge.gov.br/home/estatistica/populacao/censo2010/default.shtm, acessado em 03/ Out/2018), optou-se por calcular as taxas de ICSAP em um período único agregado, usando-se dados populacionais do Censo 2010, segundo macrorregião, sexo, faixa etária (< 1 ano, 1-4 anos) e cor/raça. Assim, as taxas referentes ao período 2009-2014 foram calculadas considerando-se o número de internações ocorridas neste período dividido por seis vezes a população de 2010, em cada segmento populacional decorrente da combinação das quatro variáveis citadas. As taxas brutas de internação foram estimadas por meio de um modelo de Poisson tendo como offset as respectivas populações. As razões de taxas para a variável cor/raça foram obtidas utilizando-se um modelo binomial negativo, uma vez que testes estatísticos evidenciaram a presença de sobredispersão nos dados analisados. A categoria branca foi usada como referência, e as taxas foram ajustadas por idade, sexo e macrorregião, No processo de imputação, utilizou-se o procedimento de imputação múltipla (Proc MI), e na análise dos modelos de Poisson e Binomial Negativo, o procedimento para modelos lineares generalizados (Proc Genmod), todos disponíveis no software SAS v9.3 (https://www.sas.com/).

\section{Resultados}

No período de 2009 a 2014 foram registradas pelo SIH/SUS 7.158 .317 hospitalizações em crianças < 5 anos, correspondendo a uma média anual de 1.193.052 internações. Apenas a variável cor/raça apresentou registros não informados. No Brasil, verificou-se 37,9\% de incompletude dessa variável durante o período acumulado de 2009-2014, com menor proporção no ano de análise mais recente (2014: 33,7\%). No segundo triênio da análise (2012-2014) foi observado um melhor preenchimento da variável cor/raça no Brasil e nas regiões. As proporções mais elevadas de cor/raça ignorada em todo o período ocorreram no Centro-oeste, em 2011 e 2012, 54,8\% e 54,1\% respectivamente, seguido pelo Nordeste (ano 2011: 50,8\%), atingindo o menor valor na Região Sul (ano 2014: 20,7\%) (dados não tabulados). 
Houve queda no número absoluto de internações no período de estudo, passando de 1.279.239, em 2009, para 1.123.440, em 2014. Essa redução foi confirmada em todas as categorias de cor/raça, na comparação dos triênios, exceto na categoria amarela, na qual houve um incremento de $15 \%$ nas internações no segundo triênio (Tabela 1). Em todas as categorias de cor/raça e em ambos os triênios verificou-se maior proporção de internações no sexo masculino. As internações foram mais frequentes na faixa etária de 1 a 4 anos no triênio 2009-2011, exceto para indígenas, em que 51,2\% das internações ocorreram em menores de um ano. No triênio seguinte, as crianças amarelas (55,8\%), brancas $(51,4 \%)$ e com cor/raça ignorada $(52,3 \%)$ passaram a ter mais internações em menores de um ano, ao passo que indígenas apresentaram proporção de internação mais elevada entre 1 e 4 anos (53,4\%). Destacam-se incrementos expressivos nas proporções de internações entre os triênios para a categoria amarela, no Nordeste, e para indígena, no Norte, além de redução expressiva para preta, amarela e indígena, respectivamente, no Nordeste, Centro-oeste e Sudeste. O tempo de permanência hospitalar mais frequente foi de 2 a 7 dias em todas as categorias de cor/raça. Os indígenas tenderam a apresentar internações com menores tempos de permanência.

As doenças do aparelho respiratório (Capítulo X da CID-10) apareceram no topo, respondendo por 37,5\% das internações em menores de cinco anos no Brasil (Tabela 2). As IRAs corresponderam a 28,2\% das internações no geral e a 75\% das internações pelo Capítulo X. Por sua vez, a P\&I correspondeu a cerca de $1 / 4$ das internações no geral e a 87,2\% das internações por IRA. Os demais capítulos com maior relevância foram, respectivamente: Capítulo I (doenças infecciosas e parasitárias: 19,3\%), Capítulo XVI (afecções originadas no período perinatal: 17,6\%), Capítulo XI (doenças do aparelho digestivo: 4,9\%) e Capítulo XIV (doenças do aparelho geniturinário: 3,6\%).

As crianças indígenas foram proporcionalmente mais acometidas por causas respiratórias (53\%), seguidas pelas crianças pretas (40,8\%), pardas $(39,3 \%)$, brancas $(38,6 \%)$ e amarelas $(31,1 \%)$. As IRA representaram $47 \%$ do total de hospitalizações indígenas, ou $88,7 \%$ das internações por causas respiratórias, superando em $57 \%$ as crianças pretas, que ficaram na segunda posição em proporção de internação por IRA. P\&I corresponderam a 43,3\% das internações indígenas, o que representa 92,1\% das admissões por IRA neste grupo. Entre as demais categorias de cor/raça, as internações por P\&I variaram de 19 a 26\% (Tabela 2). O mesmo aconteceu com as doenças infecciosas e parasitárias (Capítulo I), em que os indígenas se sobressaíram com 26\% de internações, ao passo que as proporções de internação por esta causa variaram de 14,2\% (branca) a 24,3\% (parda) entre as demais categorias de cor/raça. Hospitalizações por afecções originadas no período perinatal (Capítulo XVI) ocorreram em maior proporção nas crianças brancas (17,9\%), já as doenças do aparelho digestivo (Capítulo XI) e doenças do aparelho geniturinário (Capítulo XIV) foram mais frequentes na categoria amarela $(6,7 \%$ e $4,2 \%$, respectivamente).

A proporção de ICSAP no período de estudo foi de 34,5\% para o conjunto da população infantil. Essa proporção variou de 26,7\%, em crianças amarelas, a 44,8\%, em indígenas. Quando as ICSAP foram desagregadas por grupos de causas e categorias de cor/raça, observou-se 22,8\% e 17,8\% de internações por gastroenterites infecciosas em indígenas e pardos, respectivamente, e as crianças brancas apresentaram 9,7\% de hospitalizações por estas causas. Internações por asma foram superiores em crianças de cores preta (6,7\%) e parda (6,6\%). As pneumonias bacterianas sobressaíram nos indígenas (6,5\%) e contribuíram com menores proporções nas categorias branca (4,3\%) e amarela (2,9\%). No grupo das demais doenças pulmonares, as crianças pretas (6,3\%) apresentaram mais internações. Já no grupo de infecções do rim e do trato urinário, verificou-se maior proporção de hospitalização na categoria branca (2\%) e menor em indígenas (0,85\%).

No Brasil, a taxa mais elevada de ICSAP foi observada em crianças indígenas (97,3/1.000 crianças), seguidas por crianças de cores parda (40,0/1.000 crianças), branca (20,9/1.000 crianças), preta (16,8/1.000 crianças) e amarela (14,8/1.000 crianças) (Tabela 3). A análise por regiões e cor/raça revelou que os indígenas apresentaram as maiores taxas no Centro-oeste (337,7/1.000 crianças), Sudeste (79,8/1.000 crianças) e Norte (50,0/1.000 crianças), com destaque para a primeira, que representou a maior taxa de todas as regiões e categorias de cor/raça. Ressalta-se que a categoria branca apresentou taxas brutas mais elevadas que a preta em três regiões (Centro-oeste, Sudeste e Sul), com magnitude claramente convincente apenas na Região Centro-oeste.

De forma geral, a imputação de dados faltantes de cor/raça não modificou a direção das medidas de associação em relação às estimativas brutas (Tabela 4). As diferenças mais expressivas nas taxas de 


\section{Tabela 1}

Características demográficas e tempo de permanência hospitalar em < 5 anos, segundo categorias de cor/raça. Brasil, triênios $2009-2011$ e $2012-2014$ (Sistema de Informações Hospitalares do Sistema Único de Saúde).

\begin{tabular}{|c|c|c|c|c|c|c|c|c|c|}
\hline \multirow[b]{2}{*}{ Triênio 2009-2011 } & \multicolumn{2}{|c|}{$<5$ anos } & \multirow[b]{2}{*}{$\begin{array}{c}\text { Branca } \\
(n=1.036 .491)\end{array}$} & \multirow[b]{2}{*}{$\begin{array}{c}\text { Preta } \\
(n=64.590)\end{array}$} & \multicolumn{2}{|c|}{ Cor/Raça (\%) } & \multirow[b]{2}{*}{$\begin{array}{l}\text { Indígena } \\
(n=26.296)\end{array}$} & \multirow[b]{2}{*}{$\begin{array}{c}\text { NI } \\
(n=1.448 .921)\end{array}$} & \multirow{2}{*}{ Valor de $\mathrm{p}$ * } \\
\hline & $\mathbf{n}$ & $\%$ & & & $\begin{array}{c}\text { Parda } \\
(n=1.148 .084)\end{array}$ & $\begin{array}{c}\text { Amarela } \\
(n=11.552)\end{array}$ & & & \\
\hline Sexo & & & & & & & & & $<0,001$ \\
\hline Masculino & 2.073 .634 & 55,5 & 55,8 & 57,3 & 55,4 & 57,6 & 54,6 & 55,3 & \\
\hline Feminino & 1.662 .300 & 44,5 & 44,2 & 42,7 & 44,6 & 42,4 & 45,4 & 44,7 & \\
\hline Faixa etária (anos) & & & & & & & & & $<0,001$ \\
\hline$<1$ & 1.711 .619 & 45,8 & 48,6 & 41,4 & 40,5 & 39,5 & 51,2 & 48,2 & \\
\hline $1-4$ & 2.024 .315 & 54,2 & 51,4 & 58,6 & 59,5 & 60,5 & 48,8 & 51,8 & \\
\hline Região & & & & & & & & & $<0,001$ \\
\hline Norte & 461.748 & 12,4 & 1,9 & 5,7 & 20,6 & 4,4 & 37,3 & 13,2 & \\
\hline Nordeste & 1.184 .751 & 31,7 & 9,7 & 24,7 & 42,6 & 29,0 & 7,2 & 39,6 & \\
\hline Sudeste & 1.276 .712 & 34,2 & 49,4 & 51,5 & 28,5 & 34,4 & 13,0 & 27,4 & \\
\hline Sul & 517.114 & 13,8 & 33,8 & 15,5 & 2,0 & 12,5 & 9,6 & 9,0 & \\
\hline Centro-oeste & 295.609 & 7,9 & 5,2 & 2,6 & 6,4 & 19,7 & 32,9 & 10,8 & \\
\hline Permanência (dias) & & & & & & & & & $<0,001$ \\
\hline 1 & 528.919 & 14,2 & 16,7 & 14,7 & 11,4 & 18,6 & 5,5 & 14,6 & \\
\hline $2-7$ & 2.582 .838 & 69,1 & 65,8 & 67,9 & 75,4 & 65,0 & 79,7 & 66,4 & \\
\hline $8-14$ & 378.121 & 10,1 & 10,3 & 10,7 & 8,2 & 10,5 & 9,7 & 11,49 & \\
\hline $15-21$ & 98.955 & 2,6 & 2,9 & 2,67 & 2,0 & 2,3 & 2,17 & 2,99 & \\
\hline $22-30$ & 65.485 & 1,8 & 2,0 & 1,62 & 1,3 & 1,3 & 1,34 & 1,95 & \\
\hline$>30$ & 81.616 & 2,2 & 2,3 & 2,44 & 1,6 & 2,18 & 1,66 & 2,52 & \\
\hline Triênio 2012-2014 & $\mathbf{n}$ & $\%$ & $\begin{array}{c}\text { Branca } \\
(n=926.521)\end{array}$ & $\begin{array}{c}\text { Preta } \\
(n=54.259)\end{array}$ & $\begin{array}{c}\text { Parda } \\
(n=1.141 .129)\end{array}$ & $\begin{array}{c}\text { Amarela } \\
(n=13.244)\end{array}$ & $\begin{array}{l}\text { Indígena } \\
(n=23.426)\end{array}$ & $\begin{array}{c}\mathrm{NI} \\
(\mathrm{n}=1.263 .804)\end{array}$ & Valor de $p$ * \\
\hline Sexo & & & & & & & & & $<0,001$ \\
\hline Masculino & 1.895 .231 & 55,4 & 55,6 & 57,3 & 55,4 & 56,0 & 54,0 & 55,1 & \\
\hline Feminino & 1.527 .152 & 44,6 & 44,4 & 42,7 & 44,6 & 44,0 & 46,0 & 44,9 & \\
\hline Faixa etária (anos) & & & & & & & & & $<0,001$ \\
\hline$<1$ & 1.693 .709 & 49,5 & 51,4 & 45,3 & 45,0 & 55,8 & 46,6 & 52,3 & \\
\hline $1-4$ & 1.728 .674 & 50,5 & 48,6 & 54,7 & 55,0 & 44,2 & 53,4 & 47,7 & \\
\hline Região & & & & & & & & & $<0,001$ \\
\hline Norte & 406.527 & 11,9 & 1,5 & 6,0 & 19,1 & 3,3 & 43,4 & 12,7 & \\
\hline Nordeste & 1.028 .943 & 30,1 & 7,3 & 19,0 & 39,6 & 44,6 & 6,0 & 38,9 & \\
\hline Sudeste & 1.225 .490 & 35,8 & 50,3 & 55,8 & 31,9 & 32,2 & 6,4 & 28,4 & \\
\hline Sul & 488.699 & 14,3 & 36,0 & 16,5 & 2,4 & 11,8 & 7,4 & 9,1 & \\
\hline Centro-oeste & 272.724 & 8,0 & 4,8 & 2,6 & 7,0 & 8,1 & 36,5 & 10,8 & \\
\hline Permanência (dias) & & & & & & & & & $<0,001$ \\
\hline 1 & 540.142 & 15,8 & 19,1 & 17,1 & 13,3 & 16,6 & 5,9 & 15,6 & \\
\hline $2-7$ & 2.222 .242 & 64,9 & 61,3 & 61,4 & 69,5 & 57,3 & 78,9 & 63,3 & \\
\hline $8-14$ & 394.604 & 11,5 & 11,2 & 12,5 & 10,4 & 13,2 & 9,5 & 12,7 & \\
\hline $15-21$ & 106.083 & 3,1 & 3,3 & 3,4 & 2,6 & 6,1 & 2,6 & 3,3 & \\
\hline $22-30$ & 76.734 & 2,2 & 2,5 & 2,4 & 1,9 & 2,7 & 1,4 & 2,3 & \\
\hline$>30$ & 82.578 & 2,4 & 2,6 & 2,9 & 2,1 & 3,9 & 1,5 & 2,5 & \\
\hline
\end{tabular}

NI: não informado.

* Significância estatística do qui-quadrado, calculado com base nas diferenças entre as proporções observadas nos triênios (2009-2011 e 2012-2014), segundo categoria de cor/raça. 


\section{Tabela 2}

Hospitalização proporcional por causas em < 5 anos, segundo categorias de cor/raça. Brasil, 2009-2014.

\begin{tabular}{|c|c|c|c|c|c|c|c|c|c|c|c|c|c|c|}
\hline \multirow[t]{2}{*}{ Causas/Grupos } & \multicolumn{2}{|c|}{ Brasil } & \multicolumn{2}{|c|}{ Branca } & \multicolumn{2}{|c|}{ Preta } & \multicolumn{2}{|c|}{ Parda } & \multicolumn{2}{|c|}{ Amarela } & \multicolumn{2}{|c|}{ Indígena } & \multicolumn{2}{|c|}{ NI } \\
\hline & $\mathbf{n}$ & $\%$ & $\mathbf{n}$ & $\%$ & $\mathbf{n}$ & $\%$ & $\mathbf{n}$ & $\%$ & $\mathbf{n}$ & $\%$ & $\mathbf{n}$ & $\%$ & $\mathbf{n}$ & $\%$ \\
\hline \multicolumn{15}{|l|}{ Capítulos da CID-10 } \\
\hline$x$ & 2.677 .630 & 37,4 & 758.032 & 38,6 & 48.484 & 40,8 & 900.234 & 39,3 & 7.716 & 31,1 & 26.349 & 53,0 & 936.815 & 34,5 \\
\hline I & 1.380 .239 & 19,3 & 279.206 & 14,2 & 20.549 & 17,3 & 556.771 & 24,3 & 3.816 & 15,4 & 12.960 & 26,1 & 506.937 & 18,7 \\
\hline$X \mathrm{VI}$ & 1.259 .948 & 17,6 & 351.453 & 17,9 & 16.394 & 13,8 & 325.718 & 14,2 & 6.097 & 24,6 & 3.136 & 6,3 & 557.150 & 20,5 \\
\hline$X I$ & 351.856 & 4,9 & 102.922 & 5,2 & 6.521 & 5,5 & 103.900 & 4,5 & 1.667 & 6,7 & 926 & 1,9 & 135.920 & 5,0 \\
\hline XIV & 261.037 & 3,6 & 78.622 & 4,0 & 4.431 & 3,7 & 77.220 & 3,4 & 1.045 & 4,2 & 701 & 1,4 & 99.018 & 3,7 \\
\hline Demais capítulos & 1.227 .607 & 17,1 & 392.777 & 20,1 & 22.470 & 18,9 & 325.370 & 14,2 & 4.455 & 18,0 & 5.650 & 11,4 & 476.885 & 17,6 \\
\hline IRA * & 2.016 .082 & 28,2 & 576.508 & 29,4 & 35.473 & 29,9 & 667.086 & 29,1 & 5.497 & 22,2 & 23.380 & 47,0 & 708.138 & 26,1 \\
\hline Pneumonia \& influenza ** & 1.762 .070 & 24,6 & 491.081 & 25,0 & 29.152 & 24,5 & 595.468 & 26,0 & 4.807 & 19,4 & 21.539 & 43,3 & 620.023 & 22,9 \\
\hline \multicolumn{15}{|l|}{ ICSAP } \\
\hline Todas as causas & 2.466 .527 & 34,5 & 593.192 & 30,2 & 44.540 & 37,5 & 922.673 & 40,1 & 6.621 & 26,7 & 22.256 & 44,8 & 877.245 & 32,3 \\
\hline Gastroenterites & 956.408 & 13,4 & 191.255 & 9,7 & 13.471 & 11,3 & 406.910 & 17,8 & 2.660 & 10,7 & 11.350 & 22,8 & 330.762 & 12,2 \\
\hline Asma & 364.757 & 5,1 & 79.074 & 4,0 & 7.962 & 6,7 & 151.368 & 6,6 & 1.160 & 4,7 & 1.663 & 3,3 & 123.530 & 4,6 \\
\hline Pneumonias bacterianas *** & 364.732 & 5,1 & 83.790 & 4,3 & 6.679 & 5,6 & 133.607 & 5,8 & 730 & 2,9 & 3.229 & 6,5 & 136.697 & 5,0 \\
\hline Doenças pulmonares \# & 320.998 & 4,5 & 105.275 & 5,4 & 7.450 & 6,3 & 94.965 & 4,2 & 961 & 3,9 & 2.284 & 4,6 & 110.063 & 4,1 \\
\hline Rim e trato urinário & 129.530 & 1,8 & 40.010 & 2,0 & 1.911 & 1,6 & 38.694 & 1,7 & 278 & 1,1 & 425 & 0,9 & 48.212 & 1,8 \\
\hline Demais ICSAP & 330.102 & 4,6 & 93.788 & 4,8 & 7.067 & 6,0 & 97.129 & 4,2 & 832 & 3,3 & 3.305 & 6,7 & 127.981 & 4,7 \\
\hline
\end{tabular}

CID-10: 10ạ revisão da Classificação Internacional de Doenças ICSAP: internações por condições sensíveis à atenção primária; IRA: infecções respiratórias agudas; NI: categoria cor/raça não informada.

Fonte: lista brasileira de ICSAP 2.

Nota: capítulos da CID-10: I (doenças infecciosas e parasitárias); X (doenças do aparelho respiratório); XI (doenças do aparelho digestivo); XIV (doenças do aparelho geniturinário); XVI (doenças do período perinatal).

* CID-10: J00-J22;

** CID-10: J09-J18;

*** CID-10: J13-J139, J14-J149, J153-J154, J158-J159, J181;

\# CID-10: J20-J219, J40-J409, J41-J419, J42-J429, J43-J439, J47-J479, J44-J449.

\section{Tabela 3}

Taxas brutas de internações por condições sensíveis à atenção primária (ICSAP) em < 5 anos, e seus respectivos intervalos de $95 \%$ de confiança (IC95\%), segundo categorias de cor/raça e regiões. Brasil, 2009-2014.

\begin{tabular}{lccccc}
\hline Região & $\begin{array}{c}\text { Branca } \\
\text { Taxa (IC95\%) }\end{array}$ & $\begin{array}{c}\text { Preta } \\
\text { Taxa (IC95\%) }\end{array}$ & $\begin{array}{c}\text { Parda } \\
\text { Taxa (IC95\%) }\end{array}$ & $\begin{array}{c}\text { Amarela } \\
\text { Taxa (IC95\%) }\end{array}$ & $\begin{array}{c}\text { Indígena } \\
\text { Taxa (IC95\%) }\end{array}$ \\
\hline Norte & $9,4(9,0-9,7)$ & $14,4(13,4-15,4)$ & $55,2(54,8-55,7)$ & $8,2(6,8-9,91)$ & $50,0(48,1-52,0)$ \\
Nordeste & $12,5(12,3-12,7)$ & $12,6(12,1-13,0)$ & $49,7(49,4-49,9)$ & $14,4(13,3-15,6)$ & $16,4(14,7-18,2)$ \\
Centro-oeste & $19,5(19,1-19,8)$ & $9,9(8,9-10,9)$ & $31,1(30,6-31,6)$ & $31,2(28,3-34,5)$ & $337,7(329,5-346,1)$ \\
Sudeste & $22,0(21,8-22,1)$ & $20,2(19,6-20,7)$ & $25,5(25,2-25,7)$ & $11,8(10,8-12,9)$ & $79,8(72,5-87,7)$ \\
Sul & $30,9(30,6-31,1)$ & $28,3(26,8-29,8)$ & $12,7(12,2-13,1)$ & $17,1(14,6-20,1)$ & $32,1(28,0-36,6)$ \\
Brasil & $20,9(20,8-21,0)$ & $16,8(16,5-17,1)$ & $40,0(39,8-40,1)$ & $14,8(14,1-15,5)$ & $97,3(95,3-99,2)$ \\
\hline
\end{tabular}

Fonte: Sistema de Informações Hospitalares do Sistema Único de Saúde (SIH/SUS) e Instituto Brasileiro de Geografia e Estatística (IBGE).

Nota: taxas (por 1.000 crianças) obtidas após imputação múltipla de dados faltantes da variável cor/raça. 


\section{Tabela 4}

Razões de taxas (RT) de internações por condições sensíveis à atenção primária (ICSAP) entre categorias de cor/raça em < 5 anos, ajustadas por idade sexo e região, com seus respectivos intervalos de 95\% de confiança (IC95\%), obtidos antes e após a imputação de dados faltantes de cor/raça. Brasil e regiões, 2009-2014.

\begin{tabular}{|c|c|c|c|c|c|}
\hline & \multicolumn{5}{|c|}{ RT (IC95\%) } \\
\hline & Branca & Preta & Parda & Amarela & Indígena \\
\hline \multicolumn{6}{|c|}{ Antes da imputação } \\
\hline Norte & 1.00 & $2,03(1,67-2,48)$ & $6,39(5,27-7,76)$ & $1,00(0,81-1,24)$ & $6,11(5,02-7,44)$ \\
\hline Nordeste & 1.00 & $1,05(0,93-1,19)$ & $3,57(3,16-4,04)$ & $0,88(0,77-1,01)$ & $1,48(1,30-1,70)$ \\
\hline Centro-oeste & 1.00 & $0,39(0,34-0,45)$ & $1,45(1,29-1,62)$ & $1,48(1,30-1,68)$ & $5,70(5,08-6,4)$ \\
\hline Sudeste & 1.00 & $0,94(0,86-1,03)$ & $1,28(1,18-1,4)$ & $0,61(0,56-0,67)$ & $4,57(4,15-5,02)$ \\
\hline Sul & 1.00 & $1,12(0,98-1,28)$ & $0,44(0,39-0,51)$ & $0,71(0,61-0,82)$ & $1,34(1,17-1,55)$ \\
\hline Brasil & 1.00 & $1,01(0,73-1,40)$ & $1,71(1,23-2,38)$ & $0,88(0,64-1,23)$ & $3,35(2,42-4,67)$ \\
\hline \multicolumn{6}{|c|}{ Após a imputação } \\
\hline Norte & 1.00 & $1,76(1,46-2,11)$ & $6,37(5,39-7,53)$ & $0,96(0,75-1,24)$ & $5,95(5,00-7,07)$ \\
\hline Nordeste & 1.00 & $1,11(1,00-1,24)$ & $4,23(3,82-4,69)$ & $1,26(1,10-1,44)$ & $1,41(1,21-1,64)$ \\
\hline Centro-oeste & 1.00 & $0,57(0,49-0,66)$ & $1,70(1,52-1,90)$ & $1,76(1,52-2,05)$ & $18,51(16,53-20,73)$ \\
\hline Sudeste & 1.00 & $1,00(0,92-1,08)$ & $1,21(1,13-1,31)$ & $0,56(0,50-0,63)$ & $3,78(3,35-4,27)$ \\
\hline Sul & 1.00 & $0,99(0,88-1,12)$ & $0,44(0,39-0,49)$ & $0,60(0,49-0,72)$ & $1,06(0,90-1,26)$ \\
\hline Brasil & 1.00 & $1,00(0,68-1,47)$ & $1,91(1,31-2,80)$ & $0,94(0,64-1,39)$ & $5,75(3,93-8,42)$ \\
\hline
\end{tabular}

Fonte: Sistema de Informações Hospitalares do Sistema Único de Saúde (SIH/SUS) e Instituto Brasileiro de Geografia e Estatística (IBGE).

ICSAP antes e após a imputação foram verificadas nas crianças indígenas da Região Centro-oeste e nas amarelas da Região Nordeste. Em âmbito nacional, as crianças indígenas possuem cerca de cinco vezes a chance das crianças de cor/raça branca de internar por alguma condição sensível à atenção primária (CSAP) (RT = 5,75; IC95\%: 3,93-8,42) do que as crianças de cor/raça branca. A desagregação por região evidenciou que a razão de taxas de ICSAP entre crianças indígenas e brancas na Região Norte (RT = 5,95; IC95\%: 5,00-7,07) superou a taxa média nacional na comparação das mesmas categorias de cor/raça, atingindo um patamar ainda mais elevado na Região Centro-oeste ( RT = 18,51; IC95\%: 16,53-20,73), com razão bem superior àquela observada em análise realizada antes da imputação (RT = 5,70; IC95\%: 5,08-6,40). As crianças pardas residentes nas regiões Norte (RT = 6,37; IC95\%: 5,39-7,53) e Nordeste (RT = 4,23; IC95\%: 3,82-4,69) também se destacaram quanto à ICSAP quando comparadas às brancas. As crianças de cor/raça preta apresentaram taxa de ICSAP significativamente inferior à das brancas apenas na Região Centro-oeste (RT = 0,57; IC95\%: 0,49-0,66). Em relação às crianças amarelas, vale destacar as maiores chances observadas nas regiões Centro-oeste $(\mathrm{RT}=1,76$; IC95\%: 1,52-2,05) e Nordeste (RT = 1,26; IC95\%: 1,16-1,44), sendo que nesta última a taxa de ICSAP, entre estas crianças antes da imputação, não apresentou diferença estatisticamente significativa daquela observada entre as crianças brancas.

\section{Discussão}

Embora os registros do SIH/SUS não tenham sido planejados para a análise de situação de saúde, constituem a mais ampla fonte de dados sobre morbidade hospitalar no país 14, viabilizando a caracterização de importante dimensão da morbidade populacional, a identificação de iniquidades em saúde e a avaliação do impacto de políticas de saúde sobre as internações. Neste estudo, foi evidenciado que as hospitalizações de crianças menores de cinco anos no âmbito do SUS reduziram no período de 2009 a 2014, com destaque para a faixa etária de 1 a 4 anos. A análise das internações por causas revelou que no Brasil as doenças do aparelho respiratório, principalmente a pneumonia e influenza, respondem pela maior proporção das hospitalizações em menores de cinco anos, o que também foi verificado em todas as categorias de cor/raça. Apesar disso, verificaram-se desigualdades na proporção 
de internações por causas respiratórias e infecciosas entre categorias étnico-raciais, cujas magnitudes são mais elevadas em crianças indígenas, pretas e pardas. As doenças respiratórias $(37,4 \%)$ e as infecciosas e parasitárias $(19,3 \%)$ ocuparam o primeiro e o segundo lugares em proporção de internações no conjunto das crianças no Brasil. O mesmo foi verificado para crianças indígenas (53\% e 26,1\%), pretas $(40,8 \%$ e $17,3 \%)$ e pardas (39,3\% e $24,3 \%)$, nas quais essas duas causas respondem, em conjunto, respectivamente, por $79,1 \%, 58,1 \%$ e $63,6 \%$ das internações. Dentre as crianças brancas e amarelas, as afecções perinatais assumiram a segunda posição, à frente das causas infecciosas e parasitárias. Apenas dois estudos integrantes de uma revisão sistemática sobre causas de hospitalização em menores de cinco anos no Brasil abordam internações em âmbito nacional utilizando dados do SIH/SUS, mas nenhum descreve as internações por cor/raça, uma vez que no período investigado esta variável ainda não constava da AIH 19,20. Em um desses estudos 19, reportou-se que 40,3\% e 21,6\% das internações em crianças ocorridas entre 1998 e 2007 foram decorrentes de causas respiratórias e infecciosas e parasitárias, respectivamente, percentuais discretamente superiores aos verificados neste trabalho.

Em nossas análises, as IRA se destacaram como causas de hospitalização em crianças menores cinco anos no Brasil, respondendo por $28,2 \%$ das internações no geral e a $75 \%$ das internações pelo Capítulo X. Do mesmo modo, P\&I corresponderam a cerca de 1/4 das internações no geral e a 87,2\% das internações por IRA. A análise por cor/raça revelou predomínio de internações por IRA entre as crianças indígenas (47\%), sobretudo por P\&I (43,3\%), e as demais categorias registraram cerca de $29 \%$ (parda, branca e preta) ou menos (amarela) de internações por esta causa. Cabe ressaltar que, em 2009, houve uma pandemia causada por um novo subtipo do vírus influenza A H1N1. Naquele ano, registrou-se elevada morbidade e mortalidade por gripe entre adultos jovens e crianças menores de cinco anos 21 . No entanto, a relevância dessas causas de internação se perpetuou nos anos subsequentes, assim como já era relevante nos anos anteriores à pandemia. Fica evidente que as IRA persistem como importante agravo à saúde de crianças no Brasil, afetando principalmente grupos minoritários como os indígenas 22 .

A proporção de ICSAP também foi mais elevada dentre os indígenas, e superou em 29,8\% e 48,3\% as proporções correspondentes no Brasil e nas crianças brancas, respectivamente. As gastroenterites foram as causas mais frequentes entre as ICSAP. Diferentes doenças respiratórias também se destacaram entre as ICSAP, entretanto, na lista brasileira somente as pneumonias de origem bacteriana são consideradas CSAP. A proporção de internações por essa causa foi mais elevada entre os indígenas, tal qual evidenciado em crianças menores de cinco anos da etnia Yanomami em Roraima 13. Achados similares foram reportados na Austrália, quando comparadas as internações aborígenes e não aborígenes 23. No nosso trabalho, a hospitalização por asma foi superior nas crianças pretas e pardas, demonstrando concordância com um estudo anterior 24. Num estudo com dados nacionais do SIH/ SUS entre 1999 e 2006 25, as gastroenterites (40\%) corresponderam às principais causas de ICSAP em menores de cinco anos, resultado similar ao encontrado no nosso trabalho (39\%) nos anos mais recentes. Causas respiratórias como asma, pneumonias bacterianas e outras doenças pulmonares, também contribuíram expressivamente nas ICSAP, corroborando nossos resultados.

Dados do I Inquérito Nacional de Saúde e Nutrição dos Povos Indígenas 26, realizado entre 2008-2009, revelam que as doenças diarreicas ainda são um problema de saúde relevante em populações indígenas em todas as regiões do país, sobressaindo o Norte e o Centro-oeste, com prevalências de diarreia de $38,1 \%$ e 21,1\%, respectivamente. Essas são pelo menos 2,2 vezes maiores que a prevalência média de diarreia na população brasileira em geral $(9,4 \%) 27$ e corroboram as expressivas iniquidades verificadas nas ICSAP entre crianças indígenas e brancas no Norte e Centro-oeste do país. Alguns determinantes socioeconômicos e ambientais dessa situação são destacados na literatura, entre eles a pobreza, o saneamento básico precário, dificuldades de acesso à água potável, condições precárias de moradia, indisponibilidade de terra e restrições no acesso e qualidade da APS 26,28. Além disso, outros agravos muito comuns entre as populações indígenas costumam se sobrepor e contribuir para a manutenção do ciclo de adoecimento, tais como a desnutrição, a anemia e as IRA 26,29,30,31,32,33.

No Brasil, verificamos que a taxa de ICSAP em crianças indígenas superou em cerca de cinco vezes a taxa correspondente nas crianças brancas. As razões de taxas de ICSAP revelam alarmantes iniquidades étnico-raciais, particularmente no Norte e Centro-oeste, onde as crianças indígenas apresentaram cerca de 5 a 18 vezes a chance das crianças brancas de internar por alguma CSAP. Taxas de ICSAP em crianças Yanomami são três vezes superiores às correspondentes em crianças não indígenas de 
Roraima ou da Região Norte 13, fato que sugere que as iniquidades existem inclusive entre os povos indígenas e entre áreas de uma mesma região geográfica. A taxa de ICSAP em crianças aborígenes australianas menores que dois anos corresponde ao dobro da taxa dos não aborígenes 23 , demonstrando que as iniquidades aqui observadas também são vistas em outros países com populações nativas.

Há certo consenso internacional de que os sistemas de saúde fortemente embasados na atenção primária são mais equânimes, de modo que o monitoramento das ICSAP contribuiria para a análise de impacto relacionado à expansão de cobertura, ao acesso de grupos vulneráveis e à organizações dos serviços de saúde ${ }^{34}$. O debate acerca do uso das ICSAP como indicador de qualidade e efetividade da atenção primária vem crescendo no Brasil nos últimos anos 35,36,37,38. Grupos populacionais com limitações no acesso e qualidade da atenção à saúde tenderiam a apresentar maiores proporções e taxas de ICSAP 39. No entanto, estes autores alertam que deve haver cautela nas análises da evolução das ICSAP com vistas a discutir a efetividade da APS, pois tais indicadores sofrem efeitos dos contextos específicos, tanto relativos à organização dos serviços, quanto à cultura local. Os estudos publicados sobre ICSAP em crianças no Brasil não apresentam análises por cor/raça 3,4,5,36,40, limitando comparações com os nossos achados.

Nossos resultados são alarmantes no que tange às iniquidades reveladas nas taxas de ICSAP entre crianças indígenas e brancas no Norte e, particularmente, no Centro-oeste, assim como entre crianças pardas e brancas no Norte e Nordeste. Tais iniquidades podem ainda estar subestimadas, uma vez que muitas crianças com doença grave, particularmente nas populações mais vulneráveis e de áreas mais remotas, podem falecer antes de acessar a rede hospitalar, haja vista as altas taxas de mortalidade infantil entre indígenas e pretos 7 . Uma considerável parcela das hospitalizações infantis por IRA e diarreia, entre outros agravos, poderia ser evitada ou reduzida por meio de melhorias nas condições de vida, que vão desde a adequada nutrição, acesso e oferta oportuna e de qualidade de serviços de saúde, a ambientes de moradia satisfatórios 41 . No que se refere à situação dos povos indígenas, globalmente são reconhecidas as desigualdades nos indicadores sociais e de saúde deste segmento populacional em comparação às sociedades nacionais das quais fazem parte 42 . Nossos resultados reforçam a hipótese de que as crianças indígenas experimentam condições de vida mais precárias e limitado acesso e qualidade da APS, quando comparadas às demais crianças no Brasil.

Conforme postulado por Botelho \& Portela 39 sobre a cautela na interpretação do indicador de ICSAP, é possível supor que indivíduos em desvantagem social, como povos indígenas e outros que habitam áreas mais remotas, apresentem contextos culturais próprios e operacionalização dos serviços de saúde muito particulares, que exigem remoções para hospitais por indicações não clínicas, hipótese reforçada pelo menor tempo de permanência hospitalar verificado entre os indígenas. Isso justificaria, ao menos em parte, as iniquidades reveladas. No entanto, como citado anteriormente, as condições de vida desses povos são precárias e, possivelmente, os serviços de saúde no nível primário de atenção podem não estar disponíveis em tempo oportuno para garantir a resolubilidade da maioria das CSAP, conforme esperado na APS. Ressalta-se a que a formulação de políticas e rearranjos no SUS, particularmente a implantação do Subsistema de Atenção à Saúde Indígena, visa a enfrentar e superar possíveis cenários de iniquidades por meio da ampliação da cobertura e oferta de atenção diferenciada, adequada às demandas e especificidades locais 43 , o que parece não ter sido satisfatoriamente atingido no período estudado, não obstante os grandes avanços observados na estruturação do Subsistema de Atenção à Saúde Indígena e na ampliação da oferta e cobertura dos serviços de saúde à população indígena.

Os indicadores de ICSAP mais favoráveis encontrados para crianças pretas (Região Centro-oeste) e pardas (Região Sul) são considerados resultados inesperados e necessitam de mais investigações para sua compreensão. No entanto, possivelmente não refletem melhores condições de vida para crianças classificadas como pretas ou pardas, quando comparadas às brancas. Uma das explicações para esse achado poderia ser a excessiva restrição de acesso dessas populações aos serviços de saúde, resultando em limitada utilização da atenção hospitalar por parte das populações mais vulneráveis 44; outra possibilidade seria o oposto: essas populações poderiam estar sendo mais assistidas pelos programas de atenção primária difundidos no Brasil nos últimos anos, como a Estratégia Saúde da Família, resultando em menos ICSAP.

É importante destacar que as internações de crianças pertencentes a estratos socioeconômicos mais elevados podem estar sub-representadas nos registros do SIH/SUS, uma vez que utilizam 
serviços particulares, atenuando eventuais iniquidades nas ICSAP entre categorias de cor/raça, ao tornarem mais homogêneos do ponto de vista socioeconômico os grupos étnico raciais 34 . Dessa forma, caso a cobertura do SIH/SUS fosse mais ampla, esperaríamos potencializar as desigualdades observadas e compreender melhor esses resultados inesperados, que poderiam ser modificados. Além disso, análises ajustadas por local de residência e nível socioeconômico poderiam colaborar para a melhor compreensão dos resultados obtidos.

Verificou-se que a completude da variável cor/raça no SIH/SUS está bastante aquém dos demais sistemas de informação em saúde, tais como de Mortalidade (SIM) e Nascidos Vivos (SINASC), o que poderia afetar a análise das iniquidades 45 . As demais variáveis usadas apresentaram 100\% de completude. Para minimizar potenciais distorções na comparação das internações por cor/raça, aplicou-se a técnica de imputação múltipla de dados para estimar os dados faltantes. A expressiva discrepância das razões de taxas de ICSAP de crianças indígenas e brancas antes e após a imputação múltipla aponta para diferenciais no preenchimento da variável cor/raça, com menor grau de observância entre as crianças indígenas. Revela ainda que a desigualdade socioeconômica atua em diferentes aspectos da vida dos indivíduos, incluindo aquele referente à qualidade de suas informações nos sistemas de registros de saúde. A imputação múltipla tem sido descrita como o método mais apropriado para lidar com o problema decorrente de informações faltantes, principalmente em um contexto no qual quase $40 \%$ das informações sobre cor/raça não foram preenchidas. O descarte desse grande volume de registros pode enviesar as estimativas. Por outro lado, cabe ressaltar que o processo de imputação múltipla não está imune a problemas, podendo também resultar em estimativas enviesadas, sendo esta ocorrência maior quando o número de observações é pequeno 46,47, o que está longe de ser o caso do presente trabalho.

Problemas relacionados à confiabilidade das causas de internação são ressaltados em estudos com o SIH/SUS 14. As inconsistências se devem principalmente à baixa qualidade dos prontuários, codificação CID-10 equivocada ou falhas no faturamento. Seria pouco plausível supor que a confiabilidade das causas de internação estaria associada à cor/raça, minimizando seus impactos nas estimativas das iniquidades nas razões de taxas.

\section{Conclusões}

Neste trabalho, evidenciou-se importantes iniquidades entre os grupos étnico-raciais no tocante às causas de internação em menores de cinco anos e à internação por causas potencialmente evitáveis. Os indígenas apresentam as condições mais desfavoráveis quando comparados às demais categorias de cor/raça, com elevadas proporções de internação por pneumonia e diarreia, outras doenças infecciosas e parasitárias e CSAP, bem como taxas de ICSAP alarmantes, sobretudo quando comparadas às demais categorias de cor/raça.

Esse cenário de saúde apresenta claras repercussões sociais e econômicas para os indígenas, assim como para outras populações vulneráveis, e para o SUS, tendo em vista que as remoções hospitalares afetam a dinâmica das famílias e, por vezes, de toda a comunidade, e resultam em custos mais elevados do tratamento hospitalar. Estudos de contextos locais, particularmente em Distritos Sanitários Especiais Indígenas no Norte e no Centro-oeste, podem contribuir para uma melhor compreensão dos determinantes das ICSAP, a fim de adequar os serviços de saúde indígena às demandas efetivas da população, fazendo-se cumprir os propósitos previstos no Subsistema de Atenção à Saúde Indígena no Brasil. De todo modo, não restam dúvidas de que medidas de promoção da saúde, com a melhoria das condições de vida, saneamento e subsistência, bem como a garantia de acesso oportuno e qualificado à atenção primária à saúde, são necessárias para minimizar as internações por causas evitáveis. Tais resultados reafirmam a importância de se ter um Subsistema de Atenção à Saúde Indígena sensível às especificidades culturais e geográficas desses povos, capaz de compensar a extrema vulnerabilidade social a que estão expostos. 


\section{Colaboradores}

Y. N. Farias contribuiu na concepção deste trabalho, obtenção das bases de dados, análise, interpretação dos dados, redação e revisão crítica do conteúdo. I. C. Leite contribuiu na análise, interpretação dos dados e revisão crítica relevante do conteúdo intelectual. M. A. M. T. Siqueira contribuiu com a revisão crítica relevante do conteúdo intelectual. A. M. Cardoso contribuiu na concepção, análise, interpretação dos dados, redação e revisão crítica relevante do conteúdo intelectual.

\section{Informações adicionais}

ORCID: Yasmin Nascimento Farias (0000-00023413-0204); Iuri da Costa Leite (0000-0002-91368948); Marilda Agudo Mendonça Teixeira de Siqueira (0000-0003-4685-9817); Andrey Moreira Cardoso (0000-0002-7591-7791).

\section{Agradecimentos}

À Coordenação de Aperfeiçoamento de Pessoal de Nível Superior (Capes) pelo apoio financeiro.

\section{Referências}

1. Kerr-Pontes LR, Rouquayrol, MZ. Medida da saúde coletiva. In: Rouquayrol MZ, Almeida Filho N, organizadores. Epidemiologia \& saúde. Rio de Janeiro: Medsi; 2003. p. 37-82.

2. Alfradique ME, Bonolo PF, Dourado I, LimaCosta MF, Macinko J, Mendonça CS, et al. Internações por condições sensíveis à atenção primária: a construção da lista brasileira como ferramenta para medir o desempenho do sistema de saúde (Projeto ICSAP - Brasil). Cad Saúde Pública 2009; 25:1337-49.

3. Moura BLA, Cunha RC, Aquino R, Medina MG, Mota ELA, Macinko J, et al. Principais causas de internação por condições sensíveis à atenção primária no Brasil: uma análise por faixa etária e região. Rev Bras Saúde Mater Infant 2010; 10 Suppl 2:S83-91.

4. Barreto JOM, Nery IS, Costa MSC. Estratégia Saúde da Família e internações hospitalares em menores de 5 anos no Piauí, Brasil. Cad Saúde Pública 2012; 28:515-26.

5. Prezotto KH, Chaves MMN, Mathias TADF. Hospital admissions due to ambulatory care sensitive conditions among children by age group and health region. Rev Esc Enferm USP $2015 ; 49: 44-53$

6. Victora CG, Aquino EM, do Carmo Leal M, Monteiro CA, Barros FC, Szwarcwald CL. Maternal and child health in Brazil: progress and challenges. Lancet 2011; 377:1863-76.

7. Cardoso AM, Santos RV, Coimbra Jr. CEA. Mortalidade infantil segundo raça/cor no Brasil: o que dizem os sistemas nacionais de informação? Cad Saúde Pública 2005; 21:1602-8.

8. Chor D, Lima CRA. Aspectos epidemiológicos das desigualdades raciais em saúde no Brasil. Cad Saúde Pública 2005; 21:1586-94.

9. Matijasevich A, Victora CG, Barros AJD, Santos IS, Marco PL, Albernaz EP, et al. Widening ethnic disparities in infant mortality in southern Brazil: comparison of 3 birth cohorts. Am J Public Health 2008; 98:692-8.

10. Caldas ADR, Santos RV, Borges GM, Valente JG, Portela MC, Marinho GL. Mortalidade infantil segundo cor ou raça com base no Censo Demográfico de 2010 e nos sistemas nacionais de informação em saúde no Brasil. Cad Saúde Pública 2017; 33:e00046516.

11. Gava C, Cardoso AM, Basta PC. Infant mortality by color or race from Rondônia, Brazilian Amazon. Rev Saúde Pública 2017; 51:35.

12. Cardoso AM, Coimbra Jr. CEA, Tavares FG. Morbidade hospitalar indígena Guarani no Sul e Sudeste do Brasil. Rev Bras Epidemiol 2010; 13:21-34.

13. Caldart RV, Marrero L, Basta PC, Orellana JDY. Fatores associados à pneumonia em crianças Yanomami internadas por condições sensíveis à atenção primária na Região Norte do Brasil. Ciênc Saúde Colet 2016; 21:1597606.

14. Bittencourt SA, Camacho LAB, Leal MC. O Sistema de Informação Hospitalar e sua aplicação na saúde coletiva. Cad Saúde Pública 2006; 22:19-30. 
15. Soares Filho AM. O recorte étnico-racial nos sistemas de informações em saúde do Brasil: potencialidades para a tomada de decisão. In: Batista LE, Werneck J, Lopes F, organizadores. Saúde da população negra. 2a Ed. Brasília: Associação Brasileira de Pesquisadores/as Negros/as; 2012. p. 34-61.

16. Basnayake TL, Morgan LC, Chang AB. The global burden of respiratory infections in indigenous children and adults: a review. Respirology 2017; 22:1518-28.

17. Alison PD. Missing data. Thousand Oaks: Sage Publications; 2001.

18. Erglund P, Heeringa S. Multiple imputation of missing data using SAS. Cary: SAS Institute; 2014.

19. Oliveira BRG, Viera CS, Collet N, Lima RAG. Causas de hospitalização no SUS de crianças de zero a quatro anos no Brasil. Rev Bras Epidemiol 2010; 13:268-77.

20. Pedraza DF, Araujo EMN, Pedraza DF, Araujo EMN. Internações das crianças brasileiras menores de cinco anos: revisão sistemática da literatura. Epidemiol Serv Saúde 2017; 26:16982.

21. Bellei N, Melchior TB. H1N1: pandemia e perspectiva atual. J Bras Patol Med Lab 2011; 47:611-7.

22. Cardoso AM. A persistência das infecções respiratórias agudas como problema de Saúde Pública. Cad Saúde Pública 2010; 26:1270-1.

23. Falster K, Banks E, Lujic S, Falster M, Lynch J, Zwi K, et al. Inequalities in pediatric avoidable hospitalizations between Aboriginal and nonAboriginal children in Australia: a population data linkage study. BMC Pediatr 2016; 16:169.

24. Sousa CA, César CLG, Barros MBDA, Carandina L, Goldbaum M, Pereira JCR. Prevalência de asma e fatores associados: estudo de base populacional em São Paulo - SP, 2008-2009. Rev Saúde Pública 2012; 46:825-33.

25. Moura BLA, Cunha RC, Aquino R, Medina MG, Mota ELA, Macinko J, et al. Principais causas de internação por condições sensíveis à atenção primária no Brasil: uma análise por faixa etária e região. Rev Bras Saúde Mater Infant 2010; 10 Suppl 1:s83-91.

26. Escobar AL, Coimbra Jr. CE, Welch JR, Horta BL, Santos RV, Cardoso AM. Diarrhea and health inequity among Indigenous children in Brazil: results from the First National Survey of Indigenous People's Health and Nutrition. BMC Public Health 2015; 15:191.

27. Cunha EMGP, Barison EM. Saúde das crianças. In: Ministério da Saúde, editor. Pesquisa Nacional de Demografia e Saúde da Criança e da Mulher - PNDS 2006: dimensões do processo reprodutivo e da saúde da criança. Brasília: Ministério da Saúde; 2009. p. 171-94.

28. Raupp L, Fávaro TR, Cunha GM, Santos RV. Condições de saneamento e desigualdades de raça/cor no Brasil urbano: uma análise com foco na população indígena com base no Censo Demográfico de 2010. Rev Bras Epidemiol 2017; 20:1-15.
29. Coimbra Jr. CE, Santos RV, Welch JR, Cardoso AM, de Souza MC, Garnelo L, et al. The First National Survey of Indigenous People's Health and Nutrition in Brazil: rationale, methodology, and overview of results. BMC Public Health 2013; 13:52.

30. Leite MS, Cardoso AM, Coimbra Jr. CEA, Welch JR, Gugelmin SA, Lira PC, et al. Prevalence of anemia and associated factors among indigenous children in Brazil: results from the First National Survey of Indigenous People's Health and Nutrition. Nutr J 2013; 12:69.

31. Barreto CTG, Cardoso AM, Coimbra Jr. CEA. Estado nutricional de crianças indígenas Guarani nos estados do Rio de Janeiro e São Paulo, Brasil. Cad Saúde Pública 2014; 30:657-62.

32. Cardoso AM, Horta BL, Santos RV, Escobar AL, Welch JR, Coimbra Jr. CE. Prevalence of pneumonia and associated factors among indigenous children in Brazil: results from the First National Survey of Indigenous People's Health and Nutrition. Int Health 2015; 7:4129.

33. Ferreira AA, Santos RV, Souza JAM, Welch JR, Coimbra Jr. CEA. Anemia e níveis de hemoglobina em crianças indígenas Xavante, Brasil Central. Rev Bras Epidemiol 2017; 20:102-14.

34. Araujo C, Silva RX. Monitoramento das desigualdades raciais em saúde. In: Popolo FD, Cunha EMGP, Ribotta B, Azevedo M, organizadores. Pueblos indígenas y afrodescendientes en América Latina: dinámicas poblacionales diversas y desafíos comunes. Rio de Janeiro: ALAP Editora; 2011. p. 151-76.

35. Caldeira AP, Fernandes VBL, Fonseca WP, Faria AA. Internações pediátricas por condições sensíveis à atenção primária em Montes Claros - MG, Brasil. Rev Bras Saúde Mater Infant 2011; 11:61-71.

36. Santos LA, Oliveira VB, Caldeira AP. Internações por condições sensíveis à atenção primária entre crianças e adolescentes em Minas Gerais, 1999-2007. Rev Bras Saúde Mater Infant 2016; 16:169-78

37. Fernandes VBL, Caldeira AP, Faria AA, Rodrigues Neto JF. Internações sensíveis na atenção primária como indicador de avaliação da Estratégia Saúde da Família. Rev Saúde Pública 2009; 43:928-36.

38. Boing AF, Vicenzi RB, Magajewski F, Boing AC, Moretti-Pires RO, Peres KG, et al. Redução das internações por condições sensíveis à atenção primária no Brasil entre 1998-2009. Rev Saúde Pública 2012; 46:359-66.

39. Botelho JF, Portela MC. Risco de interpretação falaciosa das internações por condições sensíveis à atenção primária em contextos locais, Itaboraí, Rio de Janeiro, Brasil, 2006-2011. Cad Saúde Pública 2017; 33:e00050915.

40. Costa LQ, Pinto Junior EP, Silva MGC. Tendência temporal das internações por condições sensíveis à atenção primária em crianças menores de cinco anos de idade no Ceará, 2000 a 2012. Epidemiol Serv Saúde 2017; 26:51-60. 
41. World Health Organization; United Nations Children's Fund. Ending preventable child deaths from pneumonia and diarrhoea by 2025: the integrated Global Action Plan for Pneumonia and Diarrhoea (GAPPD). Geneva: World Health Organization; 2013.

42. Anderson I, Robson B, Connolly M, Al-Yaman $\mathrm{F}$, Bjertness $\mathrm{E}$, King $\mathrm{A}$, et al. Indigenous and tribal peoples' health (The Lancet-Lowitja Institute Global Collaboration): a population study. Lancet 2016; 388:131-57.

43. Fundação Nacional de Saúde. Política Nacional de Atenção à Saúde dos Povos Indígenas. 2a Ed. Brasília: Ministério da Saúde; 2002.

44. Pazó RG, Frauches DO, Maria CBM, Cade NV. Modelagem hierárquica de determinantes associados a internações por condições sensíveis à atenção primária no Espírito Santo, Brasil. Cad Saúde Pública 2014; 30:1891-902.
45. Braz RM, Oliveira PDTR, Reis AT, Machado NMS. Avaliação da completude da variável raça/cor nos sistemas nacionais de informação em saúde para aferição da equidade étnico-racial em indicadores usados pelo Índice de Desempenho do Sistema Único de Saúde. Saúde Debate 2013; 37:554-62.

46. Hardt J, Herke M, Brian T, Laubach W. Multiple imputation of missing data: a simulation study on a binary response. Open J Stat 2013; 3:370-8.

47. Jakobsen JC, Gluud C, Wetterslev J, Winkel P. When and how multiple imputation should be used for handling missing data in randomized clinical trials - a practical guide with flowcharts. BMC Med Res Methodol 2017; 17:162. 


\section{Abstract}

There has been a global increase in hospital admissions for primary care-sensitive conditions (PCSCs) as an indicator of effectiveness in primary health care. This article analyzes ethnic and racial inequalities in cause-related hospitalizations in under-five children in Brazil as a whole and the country's five major geographic regions, with an emphasis on PCSCs and acute respiratory infections (ARIs). Using data from the Hospital Information Systems of the Brazilian Unified National Health System (SIH/SUS), 2009-2014, the authors calculated proportions, rates, and rate ratios for PCSCs, adjusted by sex and age after multiple imputation of missing data on color/race. The principal causes of hospitalization were respiratory tract infections (37.4\%) and infectious and parasitic diseases (19.3\%), and indigenous children were proportionally the most affected. Crude PCSC rates (per 1,000) were highest in indigenous children (97.3; 95\%CI: 95.3-99.2), followed by brown or mixed-raced children (40.0; 95\%CI: 39.8-40.1), while the lowest rates were in Asiandescendant children (14.8; 95\%CI: 14.1-15.5). The highest adjusted rate ratios for PCSCs were seen among indigenous children compared to white children - 5.7 (95\%CI: 3.9-8.4) for Brazil as a whole, reaching 5.9 (95\%CI: 5.0-7.1) and 18.5 (95\%CI: 16.5-20.7) in the North and Central, respectively, compared to white children. ARIs remained as important causes of pediatric hospitalizations in Brazil. Alarming ethnic and racial inequalities were observed in PCSCs, with indigenous children at a disadvantage. Improvements are needed in living conditions, sanitation, and subsistence, as well as guaranteed timely access to high-quality primary health care in the more vulnerable population groups, especially the indigenous peoples of the North and Central, in order to mitigate the health inequalities and meet the guidelines of the SUS and the Brazilian Constitution.

Hospitalization; Child Health; Health of Ethnic Minorities; Health Inequity; South American Indians

\section{Resumen}

Internacionalmente, se observa un incremento en las hospitalizaciones por condiciones sensibles a la atención primaria (ICSAP), como un indicador de efectividad de la atención primaria a la salud. Este artículo analiza las inequidades étnico-raciales en las hospitalizaciones por causas evitables em menores de cinco años en Brasil y sus regiones, con énfasis en las ICSAP y en las infecciones respiratorias agudas (IRA). Con datos del Sistema de Informaciones Hospitalarias del Sistema Único de Salud (SIH/SUS), 2009-2014, se calcularon porcentajes por causas, tasas y razones de tasas de ICSAP ajustadas por sexo y edad, tras la imputación múltiple de datos faltantes de color/raza. Las principales causas de hospitalización fueron enfermedades del aparato respiratório $(37,4 \%) e$ infecciosas y parasitarias (19,3\%), siendo los niños indígenas los más afectados. Las tasas brutas de ICSAP (por 1.000) fueron más elevadas en indígenas (97,3; IC95\%: 95,3-99,2), seguidas de las mulatos/mestizos (40,0; IC95\%: 39, 8-40,1), mientras que las menores fueron en las de origen asiática (14,8; IC95\%: 14,1-15,5). Las mayores razones de tasas ajustadas de ICSAP fueron en los niños indígenas comparados a los niños de color/raza blanca - 5,7 (IC95\%: 3,9-8,4) en el país, alcanzando 5,9 (IC95\%: 5,0-7,1) y 18,5 (IC95\%: 16,5-20,7) en el Norte y Centro-oeste, respectivamente, en comparación con El color/raza blanca. Las IRA permanecen como importantes causas de hospitalización en niños em Brasil. Se observaron alarmantes in equidades étnico-raciales en las tasas de ICSAP, con situación de desventaja para los indígenas. Se necesitan mejoras en las condiciones de vida, saneamiento y subsistencia, así como la garantía de un acceso oportuno y cualificado a la atención primaria a La salud de las poblaciones más vulnerables, destacando los indígenas en el Norte y Centro-oeste, a fin de minimizar inequidades en salud y hacer cumplir las directrices del SUS y de la Constitución de Brasil.

Hospitalización; Salud del Niño; Salud de las Minorias Étnicas; Inequidad en Salud; Indios Sudamericanos
Recebido em 03/Jan/2019

Versão final reapresentada em 18/Mar/2019

Aprovado em 02/Abr/2019 